Supporting Information

\title{
Uniaxial Plasmon Coupling through Longitudinal Self- assembly of Au Nanorods
}

K. George Thomas, ${ }^{a, b^{*}}$ Said Barazzouk, ${ }^{b}$ Binil Itty Ipe, ${ }^{a}$ S. T. Shibu Joseph ${ }^{\mathrm{a}}$

and

Prashant V. Kamat ${ }^{\mathrm{b} *}$

${ }^{\mathrm{a}}$ Photosciences and Photonics Division

Regional Research Laboratory (CSIR), Trivandrum 695 019, India

and

${ }^{\mathrm{b}}$ Notre Dame Radiation Laboratory and Department of Chemical \&

Biomolecular Engineering, University of Notre Dame

Notre Dame, IN 46556-0579.

Table of contents

Page no.

1. General and Instrumentation

ii

2. Absorption spectrum of gold nanorods synthesized photochemically

ii

3. Absorption spectral changes of Au nanorods in water in the presence of MPA and effect of dodecanethiol on the spectral properties of Au nanorods

4. Effect of acetic acid and MPA on the absorption properties of Au nanorods (aspect ratio 3.3)

iv

5. Effect of MUA on the absorption properties of Au nanorods

6. TEM micrographes of gold nanorods(without zooming) 


\section{General}

Solvents and reagents used were purified and dried by standard methods. All starting materials and reagents were purchased from Sigma-Aldrich and were used as such. The electronic absorption spectra were recorded on a Shimadzu Model UV-3101 PC UV-Vis-NIR scanning spectrophotometer. TEM images were recorded on a Hitachi H 600 Electron Microscope at 75 KV.

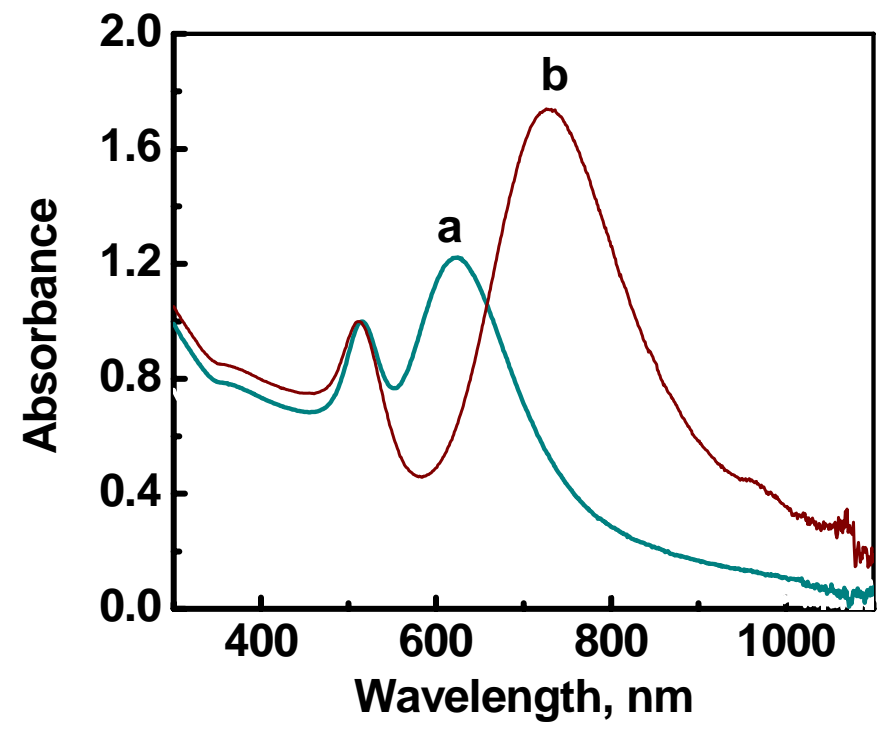

Figure S1. Absorption spectral characteristics of gold nanorods in water with two different aspect ratios. a) 3 and b) 3.3. 


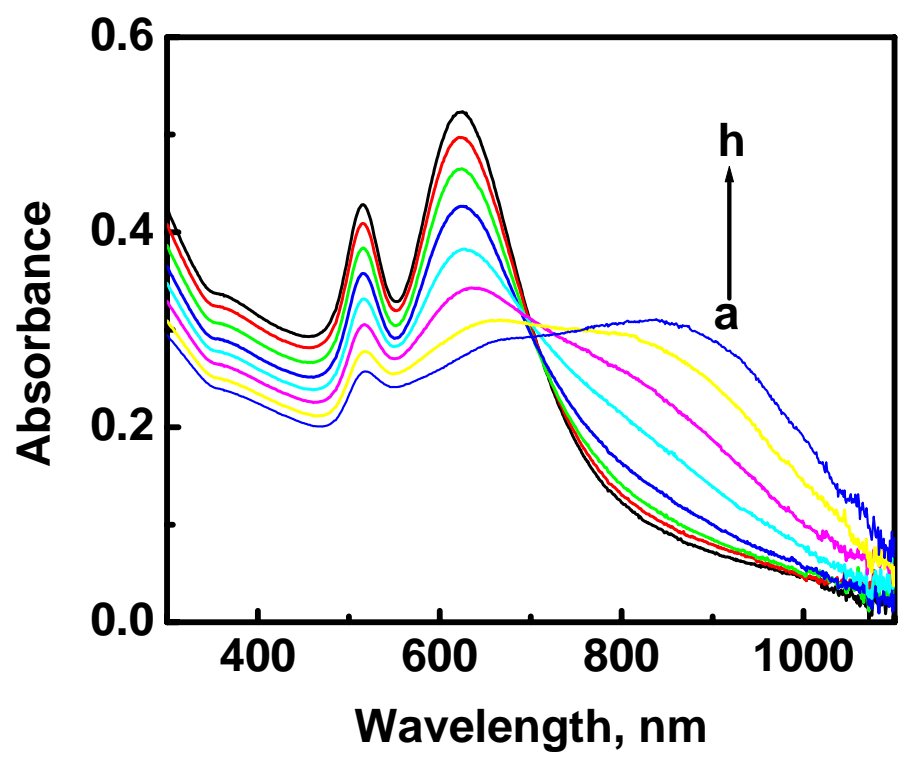

Figure S2. Absorption spectral changes of gold nanorods (aspect ratio 3) in water on addition of different concentrations of MPA. [MPA]: a) 0 $\mu \mathrm{M}$ b) $0.04 \mu \mathrm{M}$ c) $0.08 \mu \mathrm{M}$ d) $0.12 \mu \mathrm{M}$ e) $0.16 \mu \mathrm{M}$ f) $0.2 \mu \mathrm{M} \mathrm{g}) 0.24$ $\mu \mathrm{M} \mathrm{h)} 0.28 \mu \mathrm{M}$. Each spectrum was recorded 5 min after addition of MPA.

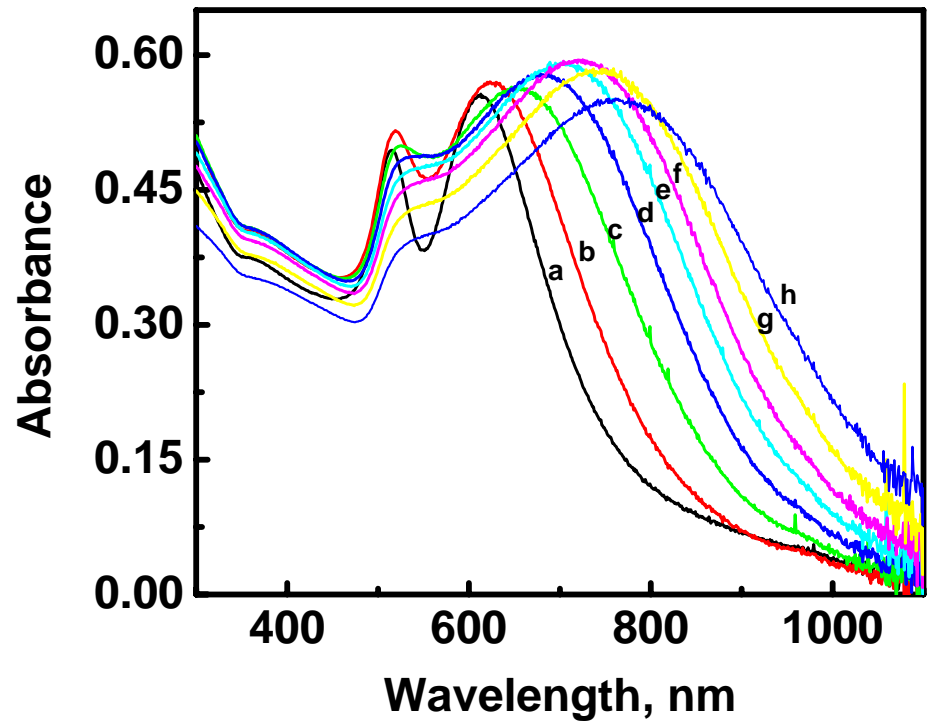

Figure S3. Absorption spectral changes of gold nanorods (aspect ratio 3) in acetonitrile-water (4:1) after adding dodecanethiol $(3 \mu \mathrm{M})$ at different time intervals. Time (min): a) 0 b) 1 c) 1.5 d) 2 e) 2.5 f) 3 g) 4 h) 7. 


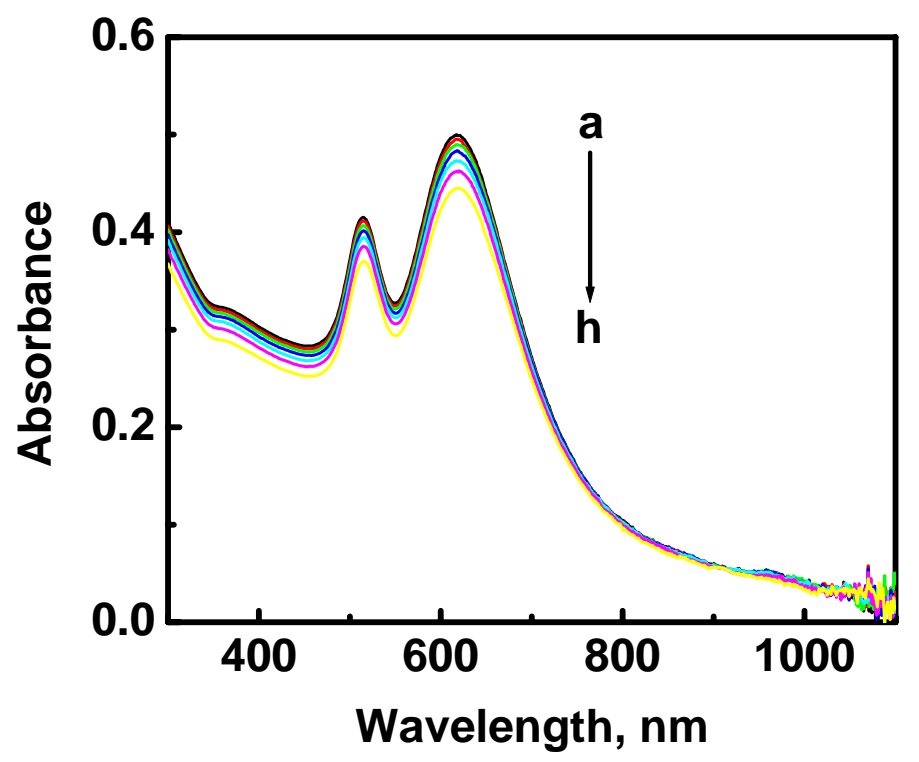

Figure S4. Absorption spectral changes of gold nanorods (aspect ratio 3) in acetonitrile-water (4:1) after adding different concentrations of glacial acetic acid. [Acetic acid]: a) $0 \mu \mathrm{M}$ b) $0.04 \mu \mathrm{M}$ c) $0.08 \mu \mathrm{M}$ d) $0.12 \mu \mathrm{M}$ e) $0.16 \mu \mathrm{M}$ f) $0.32 \mu \mathrm{M}$ g) $0.62 \mu \mathrm{M}$ h) $1.24 \mu \mathrm{M}$. Each spectrum was recorded 5 min after addition of acetic acid.

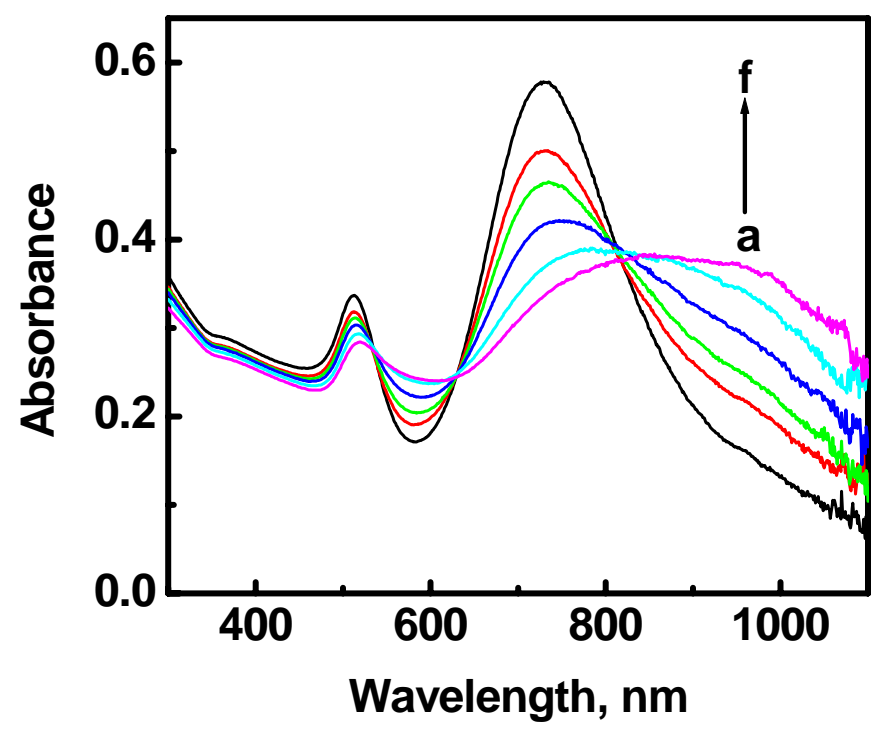

Figure S5. Absorption spectral changes of gold nanorods (aspect ratio 3.3) in acetonitrile-water (4:1) after adding different concentrations of MPA. [MPA]: a) $0 \mu \mathrm{M} \mathrm{b)} 0.4 \mu \mathrm{M}$ c) $0.6 \mu \mathrm{M} \mathrm{d}$ ) $0.8 \mu \mathrm{M}$ e) $1 \mu \mathrm{M} \mathrm{f}$ ) $1.2 \mu \mathrm{M}$. Each spectrum was recorded $5 \mathrm{~min}$ after addition of MPA. 


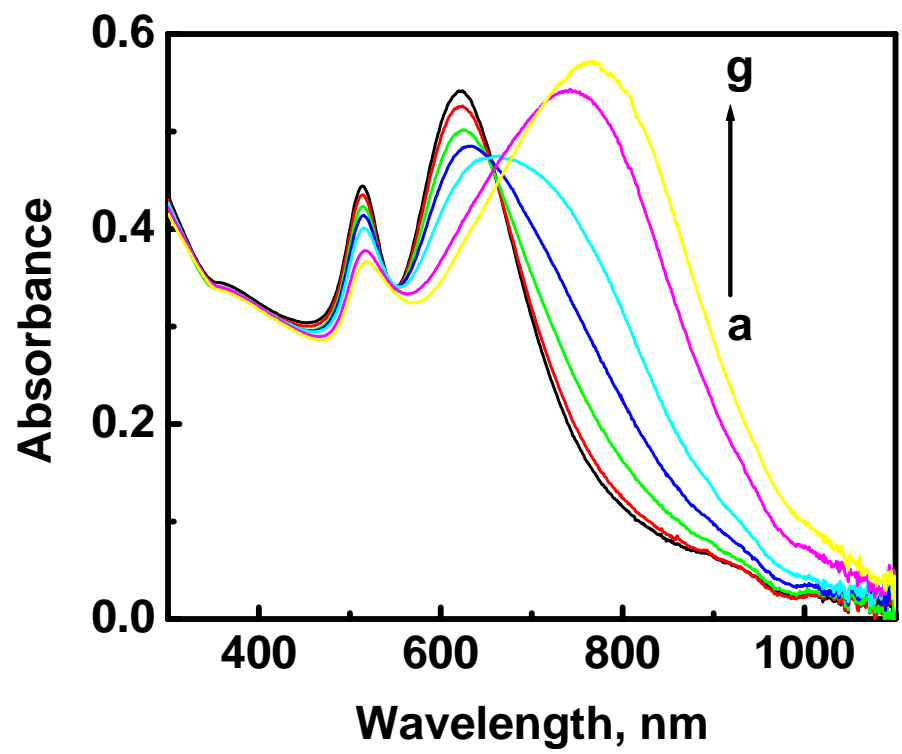

Figure S6. Absorption spectral changes of gold nanorods (aspect ratio 3.) in acetonitrile-water (4:1) after adding different concentrations of MUA. [MUA]: a) $0 \mu \mathrm{M}$ b) $0.31 \mu \mathrm{M}$ c) $0.62 \mu \mathrm{M}$ d) $0.93 \mu \mathrm{M}$ e) $1.24 \mu \mathrm{M}$ f) $1.55 \mu \mathrm{M}$ g) $1.86 \mu \mathrm{M}$. Each spectrum was recorded 5 min after addition of MUA.
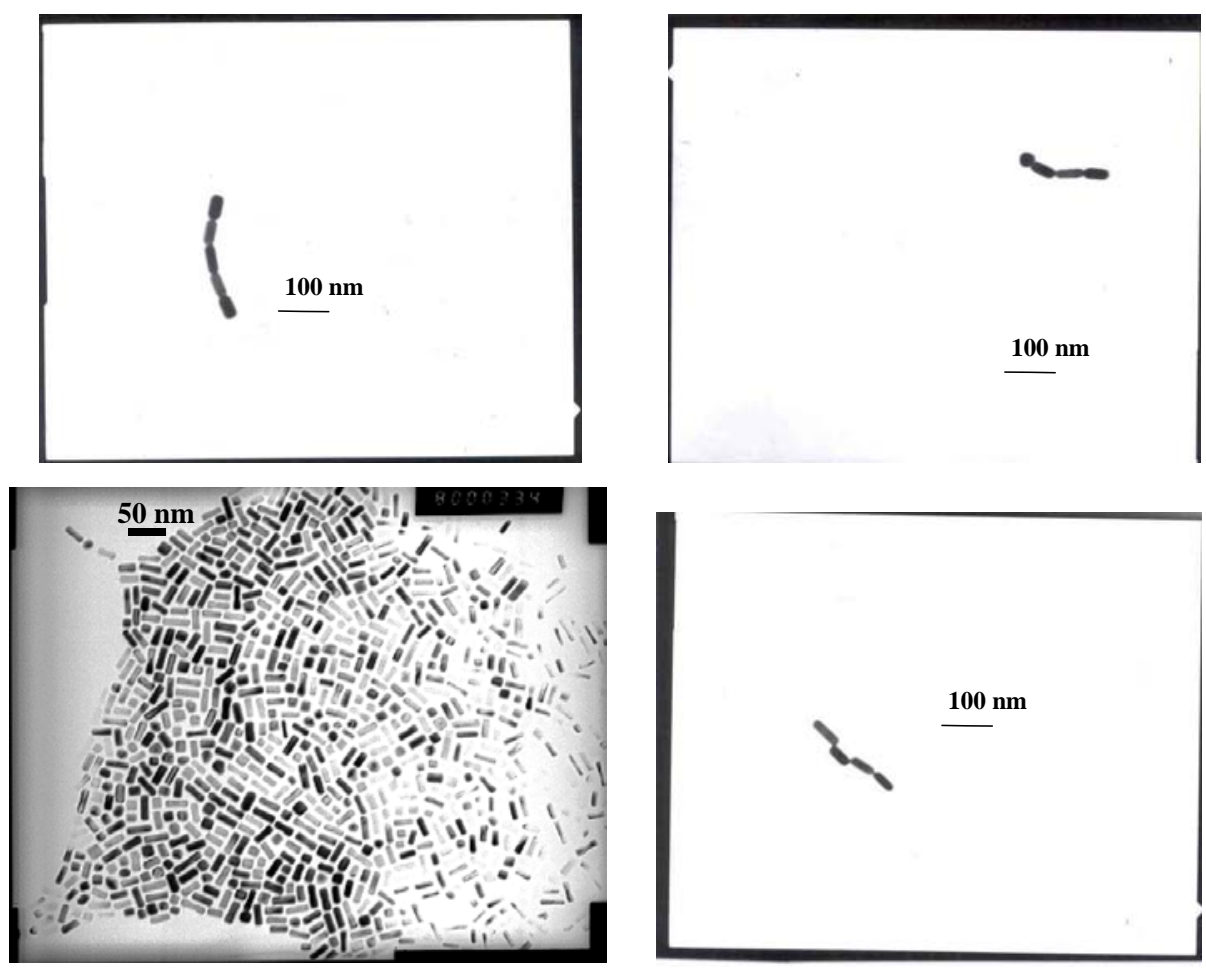

Figure S7. Unzoomed TEM micrographs of gold nanorods. 